\title{
Nível motivacional para a prática esportiva em escolares: fatores de influência
}

Motivational level for sports practice in schoolchildren: influence factors

Vinicius Machado de Oliveira

Universidade Estadual de Maringá (UEM), Brasil

oliveira_vm@hotmail.com

Marcos Roberto Brasil

Universidade Estadual de Maringá (UEM), Brasil

brasillmr@hotmail.com.br

Verônica Volski Mattes

Universidade Estadual de Maringá (UEM, Brasil

vero_edf@hotmail.com

Schelyne Ribas da Silva

Universidade Federal de Mato Grosso (UFMT), Brasil

schelys@hotmail.com

\section{Resumo:}

Este estudo teve como objetivo analisar a motivação esportiva e seus fatores de influência em escolares brasileiros do ensino fundamental II e ensino médio. Participaram do estudo 846 escolares ( $\hat{o} 439$ meninos; 우 407 meninas) com idades compreendidas entre 10 a 19 anos. Para o inquérito da motivação esportiva foi utilizado o Sport Motivation Scale (SMS). O instrumento tem como objetivo verificar os níveis de motivação intrínseca e extrínseca para o desporto, bem como casos de desmotivação. Para realização da estatística, os dados foram submetidos ao teste de Mann Whitney. Após as análises, verificou-se que escolares do sexo masculino são mais motivados que discentes do sexo feminino. Nessa mesma direção, constatou-se também que alunos mais jovens e em etapas iniciais de escolaridade apresentam escores motivacionais mais elevados que escolares mais velhos. Outro fator de influência verificado nas análises foi o ambiente de prática, em que contextos de treinamento conseguem fornecer climas motivacionais mais intensos que a escola. Por fim, modalidades de cunho coletivo se associam melhor com motivações extrínsecas enquanto práticas individuais indicam maior proximidade com motivações de natureza intrínseca.

Palavras-CHAve: Educação Física, Esporte, Escolares, Motivação Esportiva.

\begin{abstract}
:
This study aimed to analyze the sport motivation and its influence factors in Brazilian elementary and high school students. A total of 846 schoolchildren ( 18439 boys, 우 407 girls) aged 10 to 19 participated in the study. The Sport Motivation Scale (SMS) was used to survey the sport motivation. The instrument aims at verifying the levels of intrinsic and extrinsic motivation for sport, as well as cases of demotivation. For statistical purposes, the data were submitted to the Mann Whitney test. After analysis, it was found that male students are more motivated than female students. In this same direction, it was also found that younger students -and in initial stages of schooling- have higher motivational scores than older students. Another influence factor verified in the analysis was the practice environment, in which training contexts manage to provide more intense motivational climates than the school. Finally, collective modalities are better associated with extrinsic motivations while individual practices indicate greater proximity to intrinsic motivations.
\end{abstract}

KEYWORDS: Physical Education, Sport, Schoolchildren, Sport Motivation. 


\section{INTRODUÇÃO}

O esporte pode ser considerado um dos maiores legados da humanidade. Entre outras manifestações culturais, esse fenômeno social teve papel fundamental no desenvolvimento das sociedades modernas, conhecidas e partilhadas atualmente (Elias \& Dunning, 1992). De acordo com o sociólogo alemão Norbert Elias, se o ser humano não tivesse inventado o esporte e atividades similares, provavelmente a sociedade não teria alcançado o estágio de civilização hodierno. Dentre outros fatores, o esporte permitiu criar um ambiente mimético para a manifestação das pulsões humanas que, em alguma intensidade, foram suprimidas com o processo civilizador (Elias \& Dunning, 1992; Elias, 2011). Em outras palavras, por via do desporto, os indivíduos foram autorizados a externalizar seus sentimentos sem ferir com os códigos de conduta estabelecidos no seio da sociedade. Assim, o esporte surgiu como um ambiente criado de descontrole controlado das emoções, em que a tensão-excitação poderia ser produzida com o menor risco possível as pessoas (Elias \& Dunning, 1992).

Todavia, nos últimos anos, com o advento da sociedade pós-tradicional (Giddens, 2012) ou sociedade de risco (Beck, 2011), a dinâmica do movimento foi radicalizada e o esporte ganhou vários contornos. Ou seja, com o aumento dos níveis de reflexividade (Giddens, 2012), em consonância com a metamorfose do mundo (Beck, 2018), as motivações para a prática do esporte se tornaram mais polissêmicas, tendo em vista os diversos significados que os indivíduos passaram a atribuir para a realização de suas atividades motrizes. Foi diante desse cenário que, nas últimas décadas, a Psicologia em comunicação com a Educação Física direcionou esforços à compreensão dos motivos que levam os indivíduos a praticarem esportes. Mais do que apenas conhecer as razões pelas quais os indivíduos aderem ao esporte como atividade física, passatempo ou profissão, o inquérito da motivação esportiva se evidenciou como uma oportunidade para desvelar o quanto os sujeitos encontram-se conectados com um esporte e, assim, face às insuficiências, reconstruir e organizar ambientes mais motivacionais e satisfatórios para atletas ou esportistas casuais (Januário, Colaço, Rosado, Ferreira, \& Gil, 2012).

Dessa forma, reconhecida a importância da investigação da motivação esportiva, a literatura foi contemplada com uma série de empreendimentos da área da psicologia do esporte em direção a essa temática (Salselas, Gonzalez-Boto, Tuero, \& Marquez, 2007; Campos, Vigário, \& Lüdorf, 2011; Coimbra et al., 2013; Guedes \& Neto, 2013; Voser, Hernandez, Ortiz, \& Voser, 2014; Guedes \& Missaka, 2015; Souza Filho, Albuquerque, Costa, Malloy-Diniz, Costa, \& 2018; Top, Akil, \& Akyüz, 2018), em que pese a maioria dos estudos sejam endereçados ao público de atletas de esportes de rendimento. Na contramão desse tipo de pesquisa, alguns pesquisadores procuraram transportar essa ótica de investigação para dentro das escolas (Cecchini, Méndez \& Muñiz, 2002; Zahariadis, Tsorbatzoudis \& Grouios, 2005; Januário, et al., 2012; Caruzzo et al., 2017), com a intenção de tatear os perfis motivacionais de escolares e, de algum modo, trazer dados empíricos, para não só abastecer a literatura científica como também devolver respostas para a própria comunidade escolar.

Embora esses esforços sejam louváveis, está claro que existem menos pesquisas direcionadas a escola quando comparadas com contextos de treinamento, o que gera estranheza, uma vez que a escola, sobretudo por via da Educação Física, é um dos primeiros ambientes em que os indivíduos têm contato com o esporte. Portanto, conhecer os motivos que estimulam às crianças e adolescentes a prática esportiva se torna essencial, pois assim, podem-se fornecer aulas mais motivadoras e evitar experiências desagradáveis ou até mesmo prejuízos na construção da biografia de movimento dos escolares. Ademais, cabe destacar que a motivação esportiva parece ser influenciada por diferentes fatores e rastrear essas influências pode trazer potenciais respostas para a compreensão dos baixos níveis motivacionais em sujeitos em idade escolar. Dito isso, o presente estudo teve como objetivo analisar a motivação esportiva e seus fatores de influências (sexo, idade, escolaridade, tipo de modalidade, contexto de prática) em escolares do ensino fundamental II e ensino médio. 


\section{Metodologia}

O presente empreendimento se configura no âmbito dos estudos de campo do tipo transversal com incursões descritivas e quantitativas (Thomas, Nelson \& Silverman, 2007). O estudo em questão foi submetido ao comitê de Ética em Pesquisa da Universidade Estadual do Centro Oeste (Paraná/Brasil), e aprovado sob parecer 487.685/2013 - CAAE: 23127713.8.0000.0106.

Participaram do estudo 846 escolares residentes da região urbana do município de Guarapuava-ParanáBrasil, sendo 439 meninos e 407 meninas, com idades entre 10 a 19 anos. Os alunos estavam matriculados no ensino fundamental II ( $6^{\circ}$ ao $9^{\circ}$ ano) e médio ( $1^{\circ}$ ao $3^{\circ}$ ano $)$ da rede pública de ensino do município. A amostra foi constituída pela participação voluntária das escolas e escolares.

Para a realização da coleta de dados foi aplicado aos alunos o questionário Sport Motivation Scale (SMS) de Pelletier et al., (1995) com validação para a língua portuguesa de Costa et al., (2011). O instrumento em questão trata-se de um questionário constituído por 28 itens, que visa investigar os motivos que levam os indivíduos à prática esportiva. Em linhas gerais, esses itens são distribuídos e analisados em sete categorias motivacionais, sendo elas: [1] Motivação Intrínseca para Conhecer (MI-C); [2] Motivação Intrínseca para Atingirem Objetivos (MI-AO); [3] Motivação Intrínseca para Experiências Estimulantes (MI-EE); [4] Motivação Extrínseca de Regulação Externa (ME-RE); [5] Motivação Extrínseca de Introjeção (ME-I); [6] Motivação Extrínseca de Identificação (ME-ID) ; [7] Desmotivação" (DES). Como é possível perceber, o instrumento abrange questões relacionadas tanto a aspectos intrínsecos e extrínsecos da motivação, como também fatores desmotivacionais.

Em termos de mensuração das dimensões avaliadas, cabe destacar que o SMS é um questionário dimensionado em uma escala tipo Likert, em que o respondente pode sinalizar a intensidade de sua motivação em uma amplitude que varia de um a sete pontos, estando estratificado da seguinte maneira: (1) Não corresponde nada; (2 e 3) Corresponde um pouco; (4) Corresponde Moderadamente; ( 5 e 6 ) Corresponde Muito; (7) Corresponde exatamente. Uma vez demarcado o valor referente ao grau de motivação para cada um dos itens do questionário, podemos obter o valor motivacional médio para cada uma das sete escalas motivacionais. Para isso, basta apenas somar os pontos dos itens que pertencem à determinada categoria e dividir pelo número de itens atinentes à mesma. Em síntese, quanto mais os valores se aproximam dos sete pontos, maior é a motivação para a prática esportiva. Vale sublinhar que para a escala de desmotivação considera-se o inverso.

Posteriormente a aplicação do instrumento, os dados foram tabulados e submetidos as análises no software Statistical Package for Social Science (SPSS) versão 23.0. Logo após a primeira análise, identificou-se por via do teste Kolmogorov-Smirnov que os dados apresentavam comportamento assimétrico, o que indicou a necessidade de utilização da estatística não paramétrica. Portanto, em vista disso, para a comparação das variáveis entre grupos foi utilizado o teste de Mann Whitney, sendo os resultados expressos por meio de medianas e intervalos interquartílicos. Ademais, é importante sinalizar que o nível de significância adotado neste estudo foi de $\mathrm{p}<0.05$.

\section{Resultados E Discuss Ão}

Em primeiro lugar, é oportuno mencionar que antes de responder os questionários os alunos indicavam a modalidade esportiva de seu interesse, assim respondiam aos itens do SMS com base na experiência dessa prática. Dito isso, o primeiro dado que apresentamos é referente às modalidades relatadas pelos escolares. Os resultados são apresentados em frequências absolutas na figura 1 a seguir. 
Figura 1 - Modalidades esportivas relatadas pelos escolares

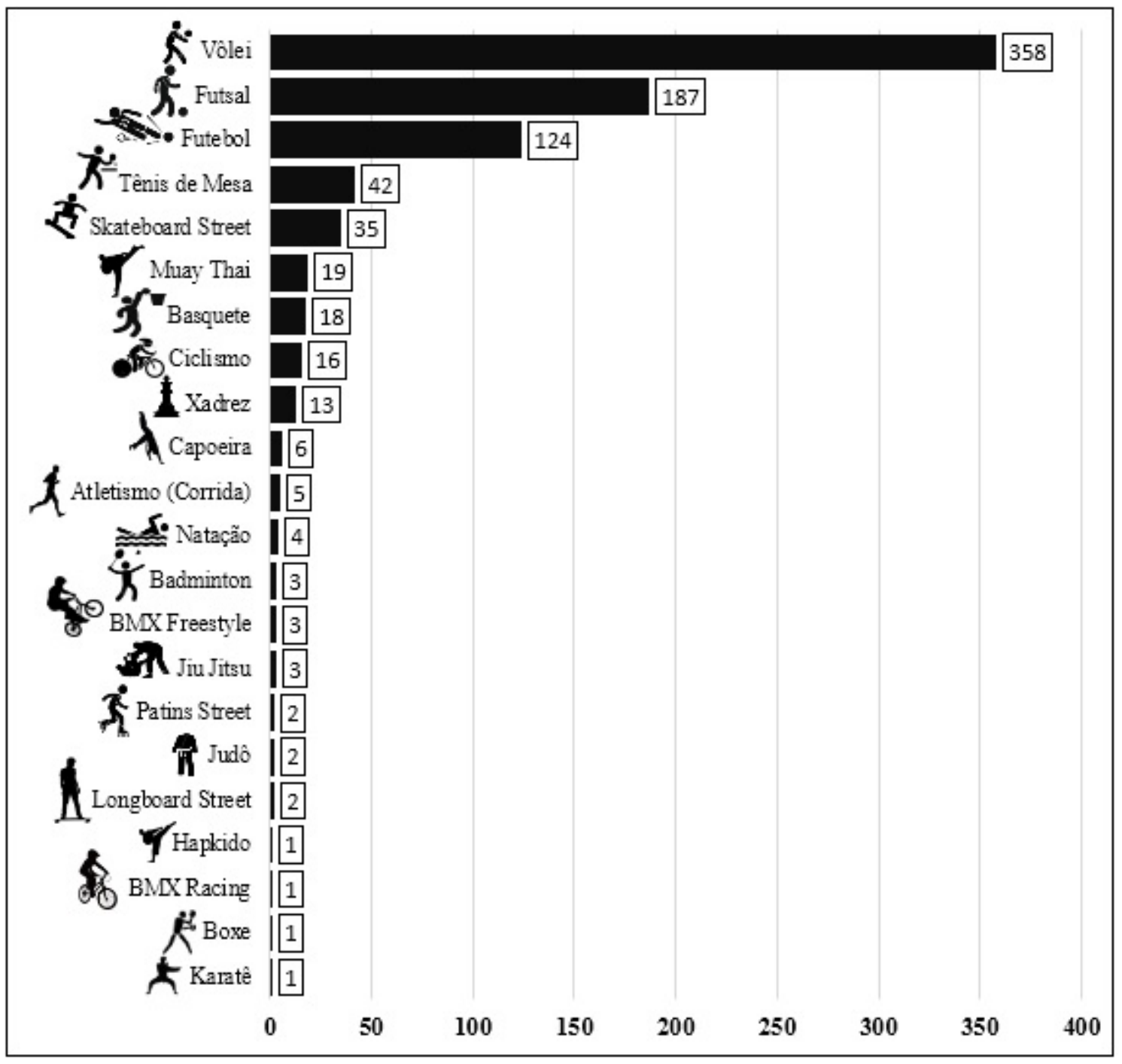

Fonte: Elaboração própria

Como podemos visualizar na referida figura, verifica-se que há uma predominância das modalidades coletivas, em detrimento as de cunho individual, sobretudo por práticas que estão enraizadas na cultura brasileira, a exemplo do voleibol, futsal e futebol, como ilustrado nos próprios dados do diagnóstico nacional do esporte (Diesporte), em que essas modalidades ainda se apresentam no rol das mais praticadas no Brasil, em especial, entre adolescentes e jovens (Brasil, 2016). Entretanto, nos últimos anos, atividades motrizes individuais têm ganhado destaque, como o caso dos esportes de aventura, musculação, lutas, trekking, dentre outras, demonstrando que, em alguma medida, está ocorrendo um processo de destradicionalização das práticas, ou seja, as pessoas estão procurando construir biografias de movimento muito diversificadas (Souza, 2019).

Em linhas gerais, o entendimento da relação do esporte com as mudanças do tecido social é fundamental, pois à medida que a sociedade se transforma as pessoas também alteram à sua maneira de se relacionar com as práticas corporais. Nesse sentido, se estamos passando por um processo de destradicionalização e individualização, como argumentam alguns intelectuais (Giddens, 2012; Beck, 2012), é natural que com o passar do tempo essa condição influencie a escolha das atividades esportivas, o que pode acarretar mudanças no quadro de hierarquia dos esportes. E, como consequência, mudanças na própria motivação para a prática 
do desporto. Todavia, para além do contexto social e escolha do esporte, há outros fatores que interferem na motivação.

$\mathrm{Na}$ sequência, procuramos observar se existem diferenças motivacionais para a prática esportiva de acordo com o sexo dos escolares. Como já dito na seção metodológica, os resultados são expressos em medianas e intervalo interquartílico (tabela 1).

Tabela 1 - Nível de motivação esportiva em relação ao sexo

\begin{tabular}{|c|c|c|c|}
\hline \multirow[b]{2}{*}{ Variáveis } & Masculino $\delta^{-7}$ & Feminino & \multirow[b]{2}{*}{$p^{-v a l o r}$} \\
\hline & $\begin{array}{l}\text { Mediana (Q1- } \\
\text { Q3) }\end{array}$ & $\begin{array}{l}\text { Mediana (Q1- } \\
\text { Q3) }\end{array}$ & \\
\hline DES & $2,25(1,50-3,50)$ & $2,50(1,50-3,50)$ & 0,198 \\
\hline ME-RE & $3,75(2,50-5,00)$ & $2,75(1,75-4,00)$ & $0,000 *$ \\
\hline ME-I & $4,50(3,25-5,50)$ & $3,75(2,75-5,00)$ & $0,000 *$ \\
\hline ME-ID & $4,25(3,25-5,25)$ & $3,50(2,50-4,75)$ & $0,000 *$ \\
\hline MI-AO & $5,00(4,00-6,00)$ & $4,00(3,00-5,25)$ & $0,000 *$ \\
\hline MI-EE & $5,25(4,25-6,25)$ & $4,25(3,00-5,50)$ & $0,000 *$ \\
\hline MI-C & $5,00(4,00-6,00)$ & $4,25(3,00-5,50)$ & $0,000 *$ \\
\hline MI & $5,00(4,25-5,92)$ & $4,17(3,17-5,25)$ & $0,000 *$ \\
\hline ME & $4,17(3,08-5,00)$ & $3,33(2,42-4,33)$ & $0,000 *$ \\
\hline
\end{tabular}

Nota: Intervalo Interquartílico (25-75), (*) Diferenças estatisticamente significativas entre grupos $(\mathrm{p} \leq 0,05)$ para o teste Mann Whitney.

Ao estratificarmos a amostra de acordo com o sexo, pôde-se constatar que os escolares do sexo masculino apresentaram escores motivacionais significativamente mais elevados para todas as dimensões analisadas. De um modo geral, os resultados indicam que os meninos demonstram maior preocupação com a aprendizagem do esporte e com o refinamento das técnicas e fundamentos; elencam-se mais emotivos quantos aos prazeres e a excitação proporcionada pela prática; possuem maiores aspirações quanto ao ganho de recompensas; importam-se mais com o julgamento e com pressões advindas de terceiros; dentre outros aspectos da motivação (Pelletier et al., 1995).

Esses dados encontrados, em alguma medida, corroboram com outras pesquisas que apontam para diferenças motivacionais em relação ao sexo (Kilpatrick, Hebert \& Bartholomew, 2005; Zahariadis et al., 2005; Januário et al., 2012; Voser et al., 2014; Andrade-Sánchez, Galindo-Villardón \& Romo, 2015; Teo et al., 2015), muito embora, é verdade, não na intensidade com que foi verificada no presente empreendimento, haja vista as discrepâncias observadas em todas as escalas. Em síntese, o que se vê com maior regularidade na literatura é que sujeitos do sexo masculino dão maior importância para a competição e para o desenvolvimento de habilidades, enquanto no lado feminino há uma maior relação com o esporte por via das relações sociais (Cecchini et al., 2002; Sirard, Pfeiffer \& Pate , 2006; Guedes \& Neto, 2013; Guedes \& Missaka, 2015).

Embora não estejam claras as razões pelas quais verificamos um nível motivacional melhor atribuído para o sexo masculino, depreende-se que esse resultado pode ter ocorrido por se tratar de uma amostra composta em sua maioria por escolares. Em outras palavras, quando meninas partilham do habitus de atleta ou participam 
de treinamentos, elas tendem a apresentar escores motivacionais muito semelhantes ou até melhores que os meninos, conforme, em alguma medida, constatado nos estudos realizados por Salselas et al., (2007) e por Campos et al., (2011). Portanto, ao que parece, quando os sujeitos estão imersos num contexto de rendimento, a influência da variável sexo é menor.

Contudo, como trata-se de escolares, outro aspecto que pode ter concorrido para uma motivação mais apática entre as meninas pode estar relacionado com o próprio nível de atividade física, pois existem vários estudos na literatura que demonstram maior prevalência de insuficiência de atividade física entre crianças e adolescentes do sexo feminino (Alberto \& Figueira Júnior; 2016; Voser, Lima, Voser, \& Duarte Junior, 2017; Silva, et al., 2018). Nessa esteira, se as meninas praticam menos atividade física, logo a aproximação com o esporte é mais esporádica e, portanto, menos motivadora para elas.

Além das diferenças entre sexo, outro fator que parece influenciar os níveis de motivação esportiva é a variável idade, como pode-se apurar nos dados a seguir (tabela 2).

Tabela 2 - Nível de motivação esportiva em relação à faixa etária

\begin{tabular}{|c|c|c|c|}
\hline \multirow[b]{2}{*}{ Variáveis } & 10 a 14 anos & 15 a 19 anos & \multirow[b]{2}{*}{$p^{-v a l o r}$} \\
\hline & $\begin{array}{l}\text { Mediana (Q1- } \\
\text { Q3) }\end{array}$ & $\begin{array}{l}\text { Mediana (Q1- } \\
\text { Q3) }\end{array}$ & \\
\hline $\mathrm{DES}$ & $2,50(1,50-3,75)$ & $2,25(1,50-3,06)$ & $0,000 *$ \\
\hline ME-RE & $3,50(2,50-4,75)$ & $2,75(1,75-4,06)$ & $0,000 *$ \\
\hline ME-I & $4,25(3,00-5,25)$ & $4,00(2,75-5,25)$ & 0,166 \\
\hline ME-ID & $4,00(3,00-5,25)$ & $3,50(2,50-4,75)$ & $0,000 *$ \\
\hline MI-AO & $4,50(3,25-5,69)$ & $4,50(3,25-5,75)$ & 0,824 \\
\hline MI-EE & $5,00(3,75-6,00)$ & $4,75(3,50-6,00)$ & 0,264 \\
\hline MI-C & $5,00(3,75-6,00)$ & $4,50(3,25-5,75)$ & $0,000 *$ \\
\hline MI & $4,83(3,83-5,67)$ & $4,58(3,50-5,58)$ & 0,076 \\
\hline ME & $4,00(3,00-4,83)$ & $3,42(2,58-4,67)$ & $0,000 *$ \\
\hline
\end{tabular}

Nota: Intervalo Interquartílico (25-75), ( $\left.{ }^{*}\right)$ Diferenças estatisticamente significativas entre grupos $(\mathrm{p} \leq 0,05)$ para o teste Mann Whitney.

Após a estratificação dos participantes em faixa-etárias, verificou-se que os indivíduos mais novos evidenciaram uma maior motivação esportiva para quase todas as subescalas analisadas, sendo os valores significativos para a ME-RE $\left(0,000^{*}\right)$, ME-ID $\left(0,000^{*}\right)$, MI-C $\left(0,000^{*}\right)$ e também para o agrupamento da ME $\left(0,000^{*}\right)$. Esses dados, em particular, sugerem que os escolares mais jovens se aproximam do esporte porque acreditam que a prática possa trazer benefícios para outros aspectos da vida cotidiana, bem como respeito, reconhecimento e prestígio (Pelletier et al., 1995).

Níveis elevados de motivação extrínseca ressaltam essa preocupação com o mundo exterior à prática, sinalizando possíveis pressões acerca da imagem perante aos outros. Em suma, trata-se de um sentimento alimentado na sociedade atual, onde há uma elevada ansiedade face às coerções e às complexidades do mundo moderno, no qual ninguém mais está safo, inclusive as crianças (Oliveira \& Souza, 2018). Em outras palavras, as crianças, desde as idades mais tenras, são estimuladas ao êxito social em seu processo de formação ou processo civilizador individual, como diria o sociólogo do esporte Norbert Elias (1993). Nesse sentido, 
compreende-se que a própria tessitura da sociedade, em alguma medida, influencia a relação das crianças com o esporte.

Ainda sobre os dados da tabela 2, visualiza-se que foi verificado um índice mais alto de desmotivação entre a faixa-etária mais nova. Em que pese não seja possível afirmar o que ocorreu, acredita-se que a própria excitação em demonstrar uma boa motivação esportiva pode ter levado as crianças e pré-adolescentes a assinalar com intensidade os itens de amotivação. Essa hipótese pode ser sustentada, uma vez que os participantes mais jovens foram os que apresentaram os maiores escores motivacionais para as outras subescalas perscrutadas. Ademais, cabe ressaltar que em ambos os grupos a escala de desmotivação foi a que apresentou os valores mais baixos quando comparada com as demais dimensóes, o que sinaliza que nos dois grupos ocorreu baixa desmotivação, muito embora a motivação dos indivíduos mais jovens se sobressaia significativamente em relação aos mais velhos.

Em resumo, se a idade parece ser um fator que repercute em níveis motivacionais diferentes - como de certa forma já bem documentado na literatura (Martínez, et al., 2008; Campos et al., 2011; Januário et al., 2012; Guedes \& Missaka, 2015) - provavelmente o nível de escolaridade pode ser outro aspecto interveniente. É justamente sobre essa inquietação que nos aprouve realizar uma comparação entre escolares matriculados no ensino fundamental versus ensino médio (Tabela 3).

Tabela 3 - Nível de motivação esportiva em relação à escolaridade

\begin{tabular}{|c|c|c|c|}
\hline \multirow{3}{*}{ Variáveis } & Fundamental & Médio & \multirow{3}{*}{ p-valor } \\
\hline & $\begin{array}{ll}\text { Mediana } & \text { (Q1- }\end{array}$ & "Mediana (Q1- & \\
\hline & Q3) & Q3) & \\
\hline DES & $2,75(1,69-4,00)$ & $2,25(1,25-3,00)$ & $0,000^{*}$ \\
\hline ME-RE & $3,75(2,50-4,75)$ & $2,75(1,75-4,00)$ & $0,000 *$ \\
\hline ME-I & $4,25(3,25-5,25)$ & $3,87(2,75-5,25)$ & $0,003 *$ \\
\hline ME-ID & $4,25(3,00-5,25)$ & $3,50(2,50-4,75)$ & $0,000 *$ \\
\hline MI-AO & $4,75(3,50-5,75)$ & $4,50(3,25-5,75)$ & 0,242 \\
\hline MI-EE & $5,00(3,94-6,00)$ & $4,62(3,25-6,00)$ & $0,009^{*}$ \\
\hline $\mathrm{MI}-\mathrm{C}$ & $5,00(3,75-6,00)$ & $4,50(3,00-5,75)$ & $0,000 *$ \\
\hline MI & $4,83(3,92-5,69)$ & $4,50(3,42-5,58)$ & $0,006^{*}$ \\
\hline ME & $4,08(3,08-4,92)$ & $3,33(2,50-4,58)$ & $0,000 *$ \\
\hline
\end{tabular}

Nota: Intervalo Interquartílico (25-75), ( $\left.{ }^{*}\right)$ Diferenças estatisticamente significativas entre grupos $(\mathrm{p} \leq 0,05)$ para o teste Mann Whitney.

Mediante a comparação entre o grau de escolaridade, constatamos que os discentes do ensino fundamental se encontram significativamente mais motivados que os alunos do colegial. Esses resultados demonstramque à medida que os sujeitos avançam no seu processo de formação escolar os níveis de motivação para o desporto são arrefecidos. Ainda que as razões para esse comportamento sejam desconhecidas, é provável que aqueles jovens, em etapas mais avançadas de escolaridade, dividam o seu tempo com mais atividades ou até mesmo possuam mais preocupaçóes e responsabilidades, por estarem se aproximando da fase adulta. Assim, o tempo para a prática de esportes ou atividades físicas fica mais restrito face a outras demandas da vida social. 
Além disso, cabe destacar que a própria organização do currículo ou ordenamento dos conteúdos da disciplina de Educação Física pode influenciar a motivação esportiva no âmbito escolar, como bem destacado no estudo de Caruzzo et al., (2017). Nessa esteira, sabendo que no ensino médio o tempo de formação é menor e os conteúdos aumentam, logo existe menos espaço para as atividades esportivas, ainda mais se levar em consideração que o esporte é apenas um dos conteúdos trabalhados nas aulas de Educação Física, em um universo de seis unidades temáticas (Esporte, Jogos e Brincadeiras, Dança, Ginastica, Lutas; Práticas Corporais de Aventura) indicadas pelos documentos normativos que orientam a disciplina no Brasil (Brasil, 1998, 2016, 2017; Paraná, 2008). Acrescenta-se a essa conformação o fato que, em diversos estados brasileiros, os alunos do colegial têm em média duas aulas semanais de Educação Física, o que restringe ainda mais a prática esportiva no contexto escolar. Talvez por isso adolescentes sintam-se menos motivados para a prática quando comparados com escolares mais jovens.

Não obstante, mesmo que identificada essas limitações do esporte na escola, os escolares relataram ter contanto com uma série de modalidades (figura 1), seja dentro ou fora dela. Em suma, práticas de caráter coletivo e individual. Com base nesses dados, realizamos uma análise comparativa entre os esportes, a fim de rastrear as possíveis diferenças entre os dois tipos de prática (tabela 4).

Tabela 4 - Nível de motivação esportiva em relação ao tipo de modalidade praticada

\begin{tabular}{llll}
\hline \multirow{2}{*}{ Variáveis } & \multicolumn{1}{l}{ Coletiva } & \multicolumn{2}{l}{ Individual } \\
\cline { 2 - 3 } & Mediana Q1-Q3) & $\begin{array}{l}\text { Mediana } \\
\text { Q3) }\end{array}$ & (Q1-valor \\
& & & \\
\hline DES & $2,50(1,50-3,50)$ & $2,25(1,25-3,50)$ & 0,099 \\
ME-RE & $3,25(2,25-4,50)$ & $3,00(1,75-4,25)$ & $0,044^{*}$ \\
ME-I & $4,25(3,00-5,25)$ & $4,00(2,75-5,00)$ & 0,205 \\
ME-ID & $4,00(2,75-5,00)$ & $3,75(2,50-5,00)$ & 0,087 \\
MI-AO & $4,50(3,25-5,50)$ & $5,25(3,75-6,00)$ & $0,002^{*}$ \\
MI-EE & $4,75(3,75-6,00)$ & $5,00(3,50-6,00)$ & 0,762 \\
MI-C & $4,75(3,50-5,75)$ & $4,75(3,50-6,00)$ & 0,159 \\
\hline MI & $4,67(3,67-5,58)$ & $4,92(3,83-6,00)$ & 0,073 \\
ME & $3,83(2,83-4,75)$ & $3,50(2,50-4,58)$ & $0,042^{*}$ \\
\hline
\end{tabular}

Nota: Intervalo Interquartílico (25-75), (*) Diferenças estatisticamente significativas entre grupos $(\mathrm{p} \leq 0,05)$ para o teste Mann Whitney.

Conforme pode-se apurar na tabela em análise, estudantes afetos a práticas coletivas se apresentam extrinsecamente mais motivados, em particular para a ME-RE $\left(0,044^{*}\right)$, quando comparados com o desporto individual. Em termos gerais, os resultados dão conta que os estudantes ligados ao desporto coletivo demonstram preocupações quanto ao ganho de reconhecimento e recompensas da prática (Standage \& Treasure; 2002; Murcia, Gimeno, \& Coll, 2007). Essa condição, por assim dizer, é previsível, uma vez que, ao partilhar de uma prática que envolve mais indivíduos, há um certo receio com a autoimagem frente aos demais. Mas não só isso, apreensões relativas à ME também sinalizam um maior estado de alerta para situações inibidoras ou de constrangimento, de modo a evitar possíveis efeitos colaterais da prática, tal como punições (Deci \& Ryan, 2000; Becker, 2002; Coimbra et al., 2013). 
Já com relação aos praticantes das modalidades individuais, identificamos uma maior sintonia com a MI, sobretudo em correspondência com a MI-AO $\left(0,002^{*}\right)$. Entre outros critérios, estar intrinsecamente mais motivado quer dizer que o indivíduo manifesta intencionalidades focadas na prática, ou seja, apresenta desejo em aprender sobre o esporte; sente prazer e satisfação em melhorar as habilidades e técnicas requeridas pela modalidade; expressa pulsões como excitação, euforia, divertimento; preocupa-se menos com as recompensas ou exterioridades da prática; e entre outros atributos (Pelletier et al., 1995; Deci \& Ryan, 2000; Keller, Tempski, Gaspar, \& Coelho 2007; Miranda \& Bara-Filho, 2008; Coimbra et al., 2013). Enfim, os resultados sugerem que os adeptos aos esportes de natureza individual conseguem estabelecer maior conexão com a prática em detrimento das dimensões externas.

Os motivos que levam os atletas a optarem por determinado estilo de esporte podem ser muitos, inclusive há ainda um vasto campo de possibilidades quanto à análise da motivação em diferentes modalidades, ainda que alguns empreendimentos já tenham fornecido resultados reveladores quanto às práticas individuais e coletivas (Murcia et al., 2007; Coimbra et al., 2013; Guedes \& Neto, 2013; Top et al., 2018). Em relação a esses estudos, cabe destacar a pesquisa de Guedes \& Neto que, ao investigar a motivação esportiva em 1517 atletas com idades entre 12 a 18 anos, encontrou relações próximas à presente pesquisa, em que indivíduos ligados à práticas coletivas preocupam-se mais com a aceitação de grupo enquanto aqueles aderentes a modalidades individuais estão mais concentrados e introspectivos ao esporte em si. Em resumo, ao que parece, esportes coletivos associam-se melhor a fatores extrínsecos e os individuais a intrínsecos.

Como se vê na tabela 4, o tipo de modalidade praticada pelos sujeitos pode determinar o tipo de motivação. Contudo, mais do que o gênero esportivo, outra variável que tende a impactar os níveis motivacionais é o contexto onde o esporte é vivenciado. Nesse sentido, ainda se valendo do banco de dados e como guisa de fechamento desta pesquisa, procuramos levar a efeito uma análise comparativa entre os ambientes de prática. Os dados são expostos na tabela 5.

Tabela 5 - Nível de motivação esportiva em relação ao ambiente de prática

\begin{tabular}{llll}
\hline \multirow{2}{*}{ Variáveis } & Escola/Outros & \multicolumn{1}{l}{ Treinamento } & \\
& & Esportivo & \multirow{2}{*}{ p-valor } \\
\cline { 2 - 3 } & Mediana (Q1-Q3) & Mediana (Q1-Q3) & \\
\hline DES & $2,50(1,50-3,50)$ & $2,25(1,25-3,75)$ & 0,182 \\
ME-RE & $3,00(2,00-4,25)$ & $4,25(2,75-5,25)$ & $0,000^{*}$ \\
ME-ID & $4,00(3,00-5,25)$ & $4,75(3,50-5,75)$ & $0,000^{*}$ \\
MI-AO & $3,75(2,75-5,00)$ & $4,25(3,50-5,50)$ & $0,000^{*}$ \\
MI-EE & $4,50(3,25-5,50)$ & $5,50(4,62-6,00)$ & $0,000^{*}$ \\
MI-C & $4,75(3,50-6,00)$ & $5,50(4,62-6,25)$ & $0,000^{*}$ \\
MI & $4,50(3,25-5,75)$ & $5,75(4,75-6,25)$ & $0,000^{*}$ \\
ME & $4,50(3,58-5,58)$ & $5,58(4,71-6,17)$ & $0,000^{*}$ \\
\hline
\end{tabular}

Nota: Intervalo Interquartílico (25-75), ( $\left.{ }^{*}\right)$ Diferenças estatisticamente significativas entre grupos $(\mathrm{p} \leq 0,05)$ para o teste Mann Whitney. 
Quanto à comparação entre os ambientes de prática, apuramos que os escolares que praticam sua modalidade em contextos de treinamento exibem níveis motivacionais estatisticamente superiores em relação aos estudantes que experienciam o esporte no âmbito da escola ou em locais públicos. Como pode-se notar nos dados, tanto a MI como a ME foram mais acentuadas para o grupo de treinamento. De modo geral, por meio do comportamento dos resultados, depreende-se que o clima motivacional produzido pelo treino sistemático incute sentimentos de maior aproximação com a modalidade esportiva. Em outros termos, se valendo das contribuições do sociólogo francês Pierre Bourdieu (1990), é possível que no prisma do esporte rendimento exista a construção de um habitus esportivo mais aflorado, haja vista que o contexto de treinamento, em tese, exerce maior força sobre as formas de pensar, agir e sentir daqueles que vivenciam com intensidade o campo esportivo.

Em suma, como constatado, existe fortes indícios de que o ambiente pode influenciar a motivação esportiva, isso porque de acordo com Vallerand (2007), os indivíduos sempre estão em processo de simbiose com o meio em que interagem. Assim, quanto mais os sujeitos imergem para dentro da lógica estrutural de um campo, mais conectados eles ficam com o espaço. Essa relação, em certa medida, foi observada no estudo de Souza Filho et al., (2018) que, ao avaliarem a motivação esportiva em 112 futebolistas, constataram que os atletas que passaram mais tempo em campo durante uma temporada foram os que evidenciaram os maiores níveis motivacionais para o desporto.

Ademais, se compararmos os dados do grupo de escolares do presente estudo com o grupo de atletas da pesquisa de Souza Filho et al., (2018) pode-se verificar que há um distanciamento grande entre a motivação esportiva no contexto de prática casual para o rendimento, o que potencializa ainda mais a hipótese de que quanto maior o contato com o campo esportivo maior o nível motivacional. Em síntese, das sete dimensões, seis se evidenciaram melhor nos atletas do estudo de Souza Filho et al., (2018) quando comparadas com o primeiro grupo de escolares de nosso estudo (DES 1,00 (1,00-1,50); ME-RE: 3,12 (2,37-4,12); ME-I 3,75 (2,50-5,50); ME-ID 4,00 (3,00-4,87); MI-AO 5,25 (4,62-6,00); MI-EE 6,00 (5,37-6,50); MI-C 5,37 $(4,37-6,25))$.

De maneira similar, outro estudo que revela maior motivação em atletas foi o proposto por Ribeiro et al., (2015), cuja investigação com 154 jogadores de handebol demonstrou amplitudes motivacionais elevadas tanto para desportistas do sexo masculino como feminino. Nessa mesma direção, o estudo de Guedes \& Missaka (2015), que analisou a motivação esportiva entre judocas, observou que quanto mais tempo em experiência e volume de treino os atletas possuíam, mais motivados eles se apresentavam. Enfim, há uma série de estudos na literatura que ajudam a sustentar a ideia de correlação entre a proximidade com o campo esportivo e bons níveis de motivação esportiva.

\section{Conclusões}

Após digressão realizada acerca dos motivos que levam escolares a prática de esportes, pudemos testificar que a motivação esportiva pode ser impactada por diferentes variáveis de influência. Em linhas gerais, fatores como sexo, idade, nível de escolaridade, gênero esportivo e ambiente de prática podem determinar a motivação esportiva, ao menos, quando tratamos de indivíduos em idade escolar. Em que pesem as intencionalidades para a prática do desporto possam ser múltiplas, ao trabalhar por meio de estratificação de grupos, algumas regularidades puderam ser observadas.

Nessa linha de raciocínio, no decorrer das análises, primeiramente verificou-se que escolares do sexo masculino tendem a ser mais motivados que estudantes do sexo feminino. Entre outras coisas, isso sugere que meninas apresentam um menor contato com o esporte ou ainda são menos estimuladas em relação à prática. Em resumo, diferenças como essas não deveriam ser tão desiguais se meninas recebessem os mesmos estímulos que os meninos para a prática esportiva. 
Durante o estudo constatou-se também que os sujeitos da faixa etária mais jovem, com escolaridades precoces, apresentaram maiores escores de motivação para o desporto, ainda que a subescala de desmotivação tenha variado significativamente entre os grupos. De modo geral, esses resultados apontam que à medida que os escolares avançam em idade e nível de formação, ocorre um distanciamento com o desporto. Essa condição acende o alerta de que a própria disciplina de Educação Física, em meio à organização curricular, arrefece o contato com o esporte conforme os alunos ingressam no ensino médio. No entanto, não só nessa última etapa escolar, mas em todo o ciclo da Educação Física na escola, a disciplina não consegue fornecer um clima motivacional suficientemente engajado quando comparado com a prática esportiva realizada no contexto de treinamento. Se refletido por esse ângulo, as aulas de Educação Física, muitas das vezes, se configuraram como a primeira via de contato dos indivíduos com o esporte, e nessa esteira, essa disciplina carrega a responsabilidade de estimular crianças e adolescentes nos primeiros passos em direção à construção de suas biografias de movimento. Por isso, a disciplina de Educação Física possui grande relevância, pois a partir dela, os escolares podem dar continuidade a sua prática de preferência fora do ambiente escolar e manter-se motivado para com o esporte.

Por fim, conclui-se que o ambiente de prática em que o indivíduo está envolto pode designar a forma com que os sujeitos manifestam a sua motivação esportiva. Mais do que isso, a própria escolha da modalidade em sua tessitura individual ou coletiva revela aproximações distintas com o desporto.

\section{REFERÊNCIAS}

Alberto, A. A. D., \& Figueira Junior, A. J. (2016). Prevalência de inatividade física em adolescentes e sua associação com variáveis socioculturais. Pensar a Prática, 19(4), 800-813. https://doi.org/10.5216/rpp.v19i4.35317

Andrade-Sánchez, A. I., Galindo-Villardón, M. P., \& Cuevas Romo, J. (2015). Análisis multivariante del perfil psicológico de los desportistas universitarios. Educación Fisica y Ciencia, 17(2). Recuperado de https://www.ef yc.fahce.unlp.edu.ar/article/view/EFyCv17n02a02

Beck, U. (2018). A metamorfose do mundo: novos conceitos para uma nova realidade. Rio de Janeiro: Zahar.

Beck, U. (2011). Sociedade de risco: rumo a uma outra modernidade. São Paulo: Ed. 34.

Beck, U. A. (2012) Reinvenção da política: rumo a uma teoria da modernização reflexiva. In A. Giddens, L. Scott, \& U. Beck (Orgs). Modernização reflexiva: política, tradição e estética na ordem social moderna (pp. 11-87). São Paulo: Editora da Unesp.

Becker, B. J. (2002). Psicologia aplicada ao treinador esportivo. Novo Hamburgo: Feevale.

Bourdieu, P. (1990). Coisas ditas. São Paulo: Brasiliense.

Brasil. (1998). Parâmetros Curriculares Nacionais. Secretaria de Educação Fundamental. Brasília: MEC. Retirado de h ttp://portal.mec.gov.br/seb/arquivos/pdf/fisica.pdf

Brasil. (2016). Diagnóstico nacional do esporte: O Perfil do Sujeito Praticante ou não de Esportes e Atividades Físicas da População Brasileira. Brasília: ME. Retirado de http://www.esporte.gov.br/diesporte/2.html

Brasil. (2017). Base Nacional Comum Curricular. Brasília: MEC. Retirado de http://basenacionalcomum.mec.gov.br/

Campos, L. T. da S., Vigário, P. dos S., \& Lüdorf, S. M. A. (2011). Fatores motivacionais de jovens atletas de vôlei. Revista Brasileira de Ciências do Esporte, 33(2), 303-317. http://dx.doi.org/10.1590/S0101-32892011000200 003

Caruzzo, N. M., Belem, I. C., Codonhato, R., Moreira, C. R., Sitoe, S. A., Rodrigues, J. C., \& Fiorese, L. (2017). Motivação para a prática de atividades esportivas em escolares da região noroeste do paraná. Corpoconsciência, 21(3), 46-54. Retirado de http://periodicoscientificos.ufmt.br/ojs/index.php/corpoconsciencia/article/view/5 683

Cecchini, J. A., Méndez, A., \& Muñiz, J. (2002). Motives for practicing sport in Spanish schoolchildren. Psicothema, 14(3), 523-531. Retrieved from http://www.psicothema.com/psicothema.asp?id=760 
Coimbra, D. R., Gomes S. S., Oliveira, H. Z., Rezende, R. A., Castro, D., Miranda, R., \& Bara Filho, M.G. (2013). Características motivacionais de atletas brasileiros. Revista Motricidade, 9(4), 64-72. http://dx.doi.org/10.606 3/motricidade.9(4).1179

Costa, V. T., Albuquerque, M. R., Lopes, M. C., Noce, F., Costa, I. T., Ferreira, R. M., \& Samulski, D. M. (2011). Validação da escala de motivação no esporte (SMS) no futebol para a língua portuguesa brasileira. Revista brasileira Educação Física e Esporte, 25(3), 537-546. http://dx.doi.org/10.1590/S1807-55092011000300015

Deci, E. L., \& Ryan, R. M. (2000). The "what" and "why" of goal pursuits: Human needs and the self-determination of behaviour. Psychological Inquiry, 11 (4), 227-268. http://dx.doi.org/10.1207/S15327965PLI1104_01

Elias, N. (1993). O processo civilizador: formação do Estado e civilização. Rio de Janeiro: Jorge Zahar.

Elias, N. (2011). O processo civilizador: uma história dos costumes. Rio de Janeiro: Jorge Zahar.

Elias, N., \& Dunning, E. (1992) A busca da excitação. Lisboa: Difel.

Giddens, A. (2012). A vida em uma sociedade pós-tradicional. In A. Giddens, L. Scott, \& U. Beck (Orgs). Modernização reflexiva: política, tradição e estética na ordem social moderna (pp. 89-166). São Paulo: Editora da Unesp.

Guedes, D. P., \& Missaka, M. S. (2015). Sport participation motives of young Brazilian judo athletes. Motriz, 21(1), 84-91. http://dx.doi.org/10.1590/S1980-65742015000100011

Guedes, D. P., \& Netto, J. E. S. (2013). Motivos para a prática de esportes em atletas jovens e fatores associados.Journal of Physical Education, 24(1), 21-31. Retirado de http://www.scielo.br/scielo.php?pid=S1983-3083201300010 0003\&script $=$ sci_abstract\&tlng $=$ pt

Januário, N., Colaço, C., Rosado, A., Ferreira, V., \& Gil, R. (2012). Motivação para a prática desportiva nos alunos do ensino básico e secundário: Influência do género, idade e nível de escolaridade. Motricidade, 8(4), 38-51. http: //dx.doi.org/10.6063/motricidade.8(4).1551

Keller, B., Tempski, R., Gaspar, D., \& Coelho, R. W. (2007). Relação entre a motivação intrínseca, o tempo de prática, treinos por semana e pretensão de ser um atleta profissional no futuro de atletas de jiu-jitsu. Lectures Educación Física y Deportes, 12(113). Retirado de https://www.efdeportes.com/efd113/pretensao-de-ser-um-atleta-profis sional-no-futuro-de-atletas-de-jiu-jitsu.htm

Kilpatrick, M., Hebert, E., \& Bartholomew, J. (2005). College students' motivation for physical activity: Differentiating men's and women's motives for sport participation and exercise. Journal of American College Health, 54(2), 87-94. https://doi.org/10.3200/JACH.54.2.87-94

Martínez, R., Molinero, O., Jiménez, R., Salguero, A., Tuero, C., \& Márquez, S. (2008). La motivación para la práctica en la iniciación al fútbol: influencia de la edad/categoría competitiva, el tiempo de entrenamiento y la relación con el entrenador. Apunts: Educación Física y Deportes, 93(3), 46-54. Retirado de http://www.revista-apunts. $\mathrm{com} / \mathrm{en} /$ library?article $=1269$

Miranda, R., \& Bara Filho, M. (2008). Construindo um atleta vencedor: uma abordagem psicofísica do esporte. Porto Alegre: Artmed.

Murcia, J. A. M, Gimeno, E. C., \& Coll, D. G. (2007). Analizando la motivación en el deporte: Un estudio a través de la teoria de la autodeterminación. Apuntes de Psicología, 25(1), 35-51. Retirado de http://www.apuntesdeps icologia.es/index.php/revista/article/view/113

Oliveira, V. M, \& Souza, J. (2018). A infância, o brincar e o jogar: reflexões a partir do referencial teórico de Norbert Elias. Educação em Revista, 34, 1-22. http://dx.doi.org/10.1590/0102-4698186748

Paraná. (2008). Diretrizes curriculares da educação básica: Educação Física. Curitiba: SEED. Retirado de http://www .educadores.diaadia.pr.gov.br/arquivos/File/diretrizes/dce_edf.pdf

Pelletier, L., Tuson, K. M., Fortier, M., Vallerand, R. J., Brière, N., \& Blais, M. R (1995). Toward a New Measure of Intrinsic Motivation, Extrinsic Motivation, and Amotivation in Sports: The Sport Motivation Scale (SMS). Journal of Sport e Exercise Psychology, 17, 35-53. https://doi.org/10.1123/jsep.17.1.35

Ribeiro, V. T., Oliveira, K. F., Moreira, C. R.; Sitoe, S. A.; Contreira, A. R., \& Vieira, L. F. (2015). Níveis de motivação e eficácia coletiva de atletas paranaenses de handebol em função do sexo. Congresso Internacional de Pedagogia do esporte. Maringá, Brasil: UEM. 
Salselas, V., Gonzalez-Boto, R., Tuero, C., \& Marquez, S. (2007). The relationship between sources of motivation and level of practice in young Portuguese swimmers. The Journal of Sports Medicine and Physical Fitness, 47(2), 228 233. Retrieved from https://www.ncbi.nlm.nih.gov/pubmed/17557064

Silva, J., Andrade, R. D., Capistrano, R., Felden, E. P. G., Lisboa, T., Andrade, A., \& Beltrame, T. S. (2018). Níveis insuficientes de atividade física de adolescentes associados a fatores sociodemográficos, ambientais e escolares. Ciência \& Saúde Coletiva, 23(12), 4277-4288. http://dx.doi.org/10.1590/1413-812320182312.30712016

Sirard, J. R., Pfeiffer, K. A., \& Pate, R. R. (2006). Motivational factors associated with sports program participation in middle school students. Journal of Adolescent Health, 38(6), 696-703. https://doi.org/10.1016/j.jadohealth .2005.07.013

Souza Filho, M. J., Albuquerque, M. R., Costa, I. T., Malloy-Diniz, L. F., \& Costa, V. T. (2018). Comparação do nível de motivação de atletas de futebol com alto e baixo tempo jogado nas partidas da categoria sub-20. Journal of Physical Education, 29, 1-10. http://dx.doi.org/10.4025/jphyseduc.v29i1.2911

Souza, J. (2019). Educação Física Reflexiva - problemas, hipóteses e programa de pesquisa. Movimento, 25, 1-15. htt ps://doi.org/10.22456/1982-8918.78269

Standage, M., \& Treasure, D. (2002). Relationship among achievement goal orientations and multidimensional situational motivation in physical education. British Journal of Educational Psychology, 72(1), 87-103. Retrieved from https://www.ncbi.nlm.nih.gov/pubmed/11916466

Teo, E., Khoo, S., Wong, R., Wee, E., Lim, B., \& Rengasamy, S. S. (2015). Intrinsic and Extrinsic Motivation Among Adolescent Ten-Pin Bowlers in Kuala Lumpur, Malaysia. Journal of Human Kinetics, 45(1), 241-251. https:// doi.org/10.1515/hukin-2015-0025

Thomas, J. R, Nelson, J. K, \& Silverman, S. J. (2007). Métodos de pesquisa em atividade física. Porto Alegre: Artmed.

Top, E., Akil, M., \& Akyüz, M. (2018). Investigation of the children's motives for participating in sports and the difficulties they experience. Acta Kinesiologica, 12(2), 36-44. Retrieved from http://actakinesiologica.com/inve stigation-of-the-childrens-motives-for-participating-in-sports-and-the-difficulties-they-experience/

Vallerand, R. J. (2007). Intrinsic and extrinsic motivation in sport and physical activity: a review and a look at the future. In G. Tenenbaum, \& E. Eklund (Ed.), Handbook of sport psychology (pp.49-83). New York: John Wiley.

Voser, R. C., Hernandez, J. A. E., Ortiz, L. F. R., \& Voser, P. E. G. (2014). A motivação para a prática do futsal: comparação entre atletas federados do sexo masculino e feminino. Revista Brasileira de Futsal e Futebol, 6(21), 196-201. Retirado de http://www.rbff.com.br/index.php/rbff/article/view/287/234

Voser, R. C., Lima, D. V.; Voser, P. E. G.; Duarte Junior, M. A. S. (2017). Mensuração do nível de atividade física de escolares da rede pública de ensino da cidade de Pelotas-rs. Revista Brasileira de Prescrição e Fisiologia do Exercício, $11(70), 820-825$. Retirado de http://www.rbpfex.com.br/index.php/rbpfex/article/view/1279

Zahariadis, P. N., Tsorbatzoudis, H., \& Grouios, G. (2005). The sport motivation scale for children: preliminary analysis in physical education classes. Perceptual and Motor Skills, 101(1), 13-54. https://doi.org/10.2466/pm s.101.1.43-54 